ger zwischendurch aufzugreifen, um bei weniger involvierten Lesern mehr Verständnis der ansonsten tadellosen Darstellung der Sachzusammenhänge zu erreichen.

Die methodischen Schwierigkeiten der Erfassung von Nichtwählern gestattet es leider bisher nicht, eine vergleichbare Untersuchung auch für dieses Teilelektorat durchzuführen, obwohl es insbesondere vor dem Hintergrund sinkender Beteiligungsraten höchst interessant wäre, Plischkes Arbeit als Referenzpunkt für die Frage heranzuziehen, wann Bürger die Entscheidung für oder gegen die Beteiligung an Wahlen fällen. Dies gilt auch deshalb, weil er nachweist, dass sich die Zunahme später Wahlentscheidungen nicht auf eine späte Mobilisierung der Wähler mit ursprünglicher Nichtwahl-Intention zurückführen lässt.

Plischkes Untersuchungen dürften über den rein politikwissenschaftlichen Kontext hinaus von großem Interesse sein, denn die Frage der Effektivität von Wahlkämpfen wird spätestens mit dem Einsatz von gezieltem „Agenda Setting“ und „Issue Framing“ auch von Kognitionspsychologen, Handlungstheoretikern und Konsumforschern in den Blick genommen.

Sonja Clasing

\title{
Wahlsystem- und Wahlverhaltensforschung: innovative Verbindung
}

Wagner, Aiko: Die Mikrofundierung von Duvergers Gesetz. Strategisches Wahlverhalten als Wirkungsweise politischer Institutionen (Studien zur Wahl-und Einstellungsforschung, Bd. 24), Nomos Verlagsgesellschaft, Baden-Baden 2013, 277 Seiten, € 44,-.

Der Titel der Dissertationsschrift von Aiko Wagner macht neugierig. Nichts Geringeres als die „Mikrofundierung von Duvergers Gesetz“ wird versprochen. Ausgangsfrage der Arbeit ist, über welche Muster des Wahlverhaltens der als „Duvergers Gesetz“ bekannte Zusammenhang zwischen der Durchlässigkeit des Wahlsystems und der Fragmentierung des Parteiensystems zustande kommt. Im Sinne des methodologischen Individualismus wird also nach der „Mikrofundierung einer Makrotheorie“ gesucht (S. 17). Nachdem Gary W. Cox bereits vor einiger Zeit die Effekte von Wahlsystemen auf Kandidaten umfassend untersucht hat ${ }^{1}$, richtet diese Arbeit den Blick auf die strategischen Anreize für Wähler. Folgerichtig wird eine Verknüpfung der Wahlsystem- und der Wahlverhaltensforschung angestrebt.

Der Autor beginnt mit einer sehr guten Wiedergabe des Forschungsstandes, die jedem nur wärmstens empfohlen werden kann, der sich insbesondere in die Wahlsystemforschung einlesen möchte (Kapitel 2). Bei den Theorien des Wahlverhaltens wird der Schwerpunkt auf den rational-choice-Ansatz gelegt, da der psychologische Effekt von Wahlsystemen ebenfalls auf Kosten-Nutzen-Abwägungen der Akteure beruht. Im dritten Kapitel geht Wagner über die bestehende Forschung hinaus, verknüpft beide Stränge überzeugend und generiert Hypothesen. Als Erklärungsfaktoren des Wahlverhaltens dienen zunächst die Parteiidentifikation, die ideologische Nähe und die Kandidateneinschätzung. Die zentrale Hy-

1 Vgl. Gary W. Cox, Making Votes Count. Strategic Coordination in the World's Electoral Systems, Cambridge 1997. 
pothese lautet, dass der Einfluss der ideologischen Nähe einer Partei auf das Wahlverhalten abnimmt, je höher die Wahrscheinlichkeit ist, dass eine Partei die durch das Wahlsystem erzeugte Repräsentationshürde überwinden kann (S. 107). Außerdem wird angenommen, dass Föderalismus, Präsidentialismus und Wahlsystemreformen das Zutreffen der Haupthypothese abschwächen.

Die empirische Analyse basiert auf Daten der Comparative Study of Electoral Systems (CSES), einer Sammlung von Wahlstudien aus zahlreichen demokratischen Ländern. Die wie immer diffizile Operationalisierung der Wahlsysteme stellt der Autor in Text und Anhang umfassend dar und betrachtet dabei auch komplexe Wahlsysteme aus der Perspektive eines Wählers, der seine Stimme nicht verschwenden möchte. Sodann werden Makroanalysen durchgeführt, um die grundsätzliche Existenz der mechanischen und psychologischen Effekte von Wahlsystemen aufzuzeigen. Darauf folgt die Überprüfung der Hypothesen auf Mikroebene. Die Operationalisierung der zentralen Variable - der durch das Wahlsystem bedingten Chance einer Partei, ins Parlament einzuziehen - stellt eine der Stärken der Arbeit dar (S. 159 ff.). Basierend auf der Differenz von Parteistärke und Sperrklausel wird durch eine logistische Funktion ein Chancenindex erstellt, womit der angenommene Interaktionseffekt von ideologischer Nähe und wahlsystembedingter Erfolgschance modelliert werden kann. Aufgrund der Mehrebenenstruktur der Daten - mehrere Parteipräferenzen befinden sich innerhalb von Befragten, Wahlkreisen und Ländern - werden konditionale logistische Regressionen mit korrigierten Fehlertermen geschätzt. Bei der gesamten Analyse demonstriert Wagner einen souveränen Umgang mit statistischen Methoden. Auch wird die Vorgehensweise stets transparent gemacht und nachvollziehbar begründet.

In knapp 60 Prozent der Wahlstudien kann der Interaktionseffekt in der theoretisch angenommenen Richtung nachgewiesen werden. Da bei logistischen Regressionen Koeffizienten nicht direkt verglichen werden können, erfolgt eine Umrechnung in ein verzerrungsfreies Maß, das den Grad des Zutreffens der Hypothese anzeigt. Bei der Überprüfung des Einflusses von Föderalismus, Präsidentialismus und Wahlsystemreformen zeigt sich, dass nur ersterer die Haupthypothese signifikant abschwächt. Zuletzt testet Wagner, ob politisch versierte Wähler in geringerem Maße strategisch wählen, was sich nicht bestätigt. Im Fazit fasst er seine Arbeit prägnant zusammen und zeigt Perspektiven für die weitere Forschung auf.

Wagner ist insgesamt ein großer Wurf gelungen. Es verbleiben nur einige, weniger bedeutsame Kritikpunkte: Terminologisch wäre es günstiger gewesen, von Duvergers Hypothesen zu sprechen, da Duverger drei verschiedene Behauptungen aufstellte, die sich nicht alle in ein gemeinsames „Gesetz“ überführen lassen. Absolute Mehrheitswahlsysteme funktionieren nach einer anderen, auf Allianzenbildung basierenden Logik. Die Studie untersucht auch Wahlsysteme, die Allianzen begünstigen, berücksichtigt dies aber nicht konsequent in der Operationalisierung. Während für Italien Erfolgshürde und Wahlverhalten jeweils für Parteien ermittelt wurden, wurde für andere Länder die Erfolgshürde Parteien zugeordnet, obwohl sie sich nur auf Allianzen bezieht. Das betrifft zum Beispiel Chile und die Schweiz, wo die Wahlkreisgrößen eben keinen Anreiz zur Wahl großer Parteien erzeugen, solange die präferierte kleine Partei in einer chancenreichen Allianz antritt. Ähnlich können Wähler chancenloser Kandidaten in Australien und Irland ihre Stimme übertragen.

Dass die Validierung des Makrozusammenhangs auf Ebene der Staaten theorieinadäquat ist und stattdessen auf Ebene der Wahlkreise erfolgen sollte, macht der Autor am Ende des Kapitels selbst deutlich (S. 152). Besonders die Überprüfung strategischen Kandidaturver- 
haltens wirkt dadurch wenig überzeugend. Wenn man schon die nationalen Mittelwerte der Wahlkreisgrößen benutzt, müsste auch die Parteienfragmentierung durch den Durchschnitt der Fragmentierung in den Wahlkreisen gemessen werden. Da dieses Kapitel für den Hauptargumentationsgang aber nebensächlich ist, wird die eigentliche Leistung der Arbeit nicht geschmälert. Diese besteht in der konzisen Analyse der Mikrozusammenhänge und der überzeugenden Verknüpfung zweier bislang viel zu isolierter Forschungsgebiete.

Insgesamt legt Aiko Wagner eine sehr gelungene Studie vor, die der Wissenschaft noch lange Zeit Inspiration für weitere Projekte liefern wird. Dem Autor kann nur empfohlen werden, seine Arbeit ins Englische übersetzen zu lassen, um die Erkenntnisse auch der internationalen Politikwissenschaft zugänglich zu machen.

Philipp Weinmann

\section{Die Linke in Deutschland: facettenreiches Porträt, lohnende Lektüre}

Oppelland, Torsten und Hendrik Träger: Die Linke. Willensbildung in einer ideologisch zerstrittenen Partei (Reihe "Die politischen Parteien der Bundesrepublik Deutschland“), Nomos Verlagsgesellschaft, Baden-Baden 2014, 263 Seiten, € 19,90.

In Zeiten des entideologisierten, unterschiedsarmen Pragmatismus hat ein Buch über die Linke als letzter „ideologischer“ Partei seinen besonderen Reiz. Was indes solch eine Partei dafür bezahlt, nämlich ideologische Zerstrittenheit, wird von Torsten Oppelland und Hendrik Träger als Untertitel ihres Buches gleich mitgeliefert.

Der einleitenden typologischen Charakteristik und Einordnung Der Linken folgt die Darstellung ihrer historischen und programmatischen Entwicklung, sodann die der inneren Willensbildungsprozesse und endet schließlich bei ihrem Wählerpotenzial und ihren strategischen Optionen. Das Besondere der Linken machen die beiden Autoren am typologischen Profil der Partei fest. Sie bildet einen Hybrid, der aus einer Mixtur aus Weltanschauungs-, Sammlungs-, Klassen- und Regionalpartei konstituiert wird. Dass gerade auf diese hoch interessante Kombination von Parteitypen, denen später sogar noch die Volkspartei beigefügt wird, ohne gründlichere Herleitung zurückgegriffen wird, könnte von dem einen oder anderen Kenner der typologischen Parteienforschung moniert werden.

Die Leitfrage lautet, „wie Die Linke mit diesem diffusen Charakter, mit ihrer großen innerparteilichen Heterogenität und den verschiedenen historisch-kulturellen Traditionen umgeht und wie die innerparteiliche Willensbildung in einer solchen Partei funktioniert" (S. 16).

Bei der Darstellung der historischen und programmatischen Entwicklung Der Linken gehen Oppelland und Träger bis zur 1914 beginnenden Abspaltung der USPD von der SPD und der 1919 gegründeten KPD zurück, um dieses Schisma in eine Linie mit der Abspaltung der WASG im Gefolge der Schröderschen Agenda-Reformen von 2003 zu stellen. Auch bei den Ausführungen zur SED geht es ihnen in erster Linie um das Verhältnis zur SPD, um einen Bogen zur Linken zu schlagen - ziemlich viel SPD, wo es doch um eine Genealogie der PDS/Die Linke geht und auch darum, wie Die Linke Vergangenheitsbewäl- 